\title{
Use of the National Diabetes Data Group and the Carpenter-Coustan criteria for assessing gestational diabetes mellitus and risk of adverse pregnancy outcome
}

\author{
Mei-Chun Lu' ${ }^{1+}$, Song-Shan Huang ${ }^{2+}$, Yuan-Horng Yan ${ }^{1,3,4,5}$ and Panchalli Wang ${ }^{2^{*}}$
}

\begin{abstract}
Background: The influence of different diagnostic thresholds for gestational diabetes mellitus (GDM) on pregnancy outcomes is not fully understood. Degrees of glucose intolerance according to the Carpenter-Coustan (CC) criteria were less severe than the National Diabetes Data Group (NDDG) criteria for GDM. Recent studies have shown inconsistent results regarding the risk of adverse pregnancy outcomes between the NDDG and CC criteria. Therefore, the objective of this study was to investigate whether pregnant women who met only the CC criteria but not the NDDG criteria and those who met the NDDG criteria had increased risks of adverse pregnancy outcomes compared to a negative screening group.
\end{abstract}

Methods: A total of 11,486 Taiwanese pregnancies were enrolled in a retrospective cohort study. The study subjects were classified as follows: (1) negative screening group: women with negative 50-g glucose challenge test (GCT) results, (2) false-positive screening group: women with positive GCT results and negative 100-g OGTT results according to both CC and NDDG criteria, (3) CC-only-GDM group: women with positive GCT results plus GDM diagnosis meeting the CC but not the NDDG criteria, and (4) NDDG-GDM group: women diagnosed with GDM using the NDDG criteria. Multiple mixed effects logistic regression analysis was used to examine the relationships between the groups and pregnancy outcomes.

Results: There were 9002 (78.4\%), 1776 (15.5\%), 251 (2.2\%), and 457 (4.0\%) study pregnancies in the 4 groups. Compared with the negative screening group, the maternal outcomes were not different within groups except for gestational hypertension/preeclampsia. For neonatal outcomes, the CC-only-GDM group had significantly greater risks of macrosomia, low birth weight, and admission to a neonatal intensive care unit [adjusted odds ratio (aOR), (95\% confidence interval, Cl): 2.73 (1.18-6.31), 1.64 (1.01-2.64), and 1.61 (1.05-2.46), respectively]. The NDDG-GDM group also showed significantly greater risks, and the false-positive screening group showed no differences from the negative screening group.

Conclusion: Women who met only the CC criteria and women who met NDDG criteria had significant increased risks of adverse neonatal outcomes. This evidence adds important information to the current debate about the diagnostic criteria for GDM regarding pregnancy outcomes.

Keywords: Carpenter-Coustan criteria, National Diabetes Data Group criteria, Adverse pregnancy outcome, (Continued on next page)

\footnotetext{
* Correspondence: cych10613@gmail.com

${ }^{\dagger}$ Equal contributors

${ }^{2}$ Department of Obstetrics and Gynecology, Ditmanson Medical Foundation

Chia-Yi Christian Hospital, 539 Chung-Shau Road, Chia-Yi City 600, Taiwan

Full list of author information is available at the end of the article
} 
(Continued from previous page)

Gestational diabetes mellitus, Macrosomia, Admission to a neonatal intensive care unit

Abbreviations: ACOG, The American College of Obstetricians and Gynecologists; BMI, Body mass index; CC, The Carpenter-Coustan criteria; Cl, Confidence interval; GCT, Glucose challenge test; GDM, Gestational diabetes mellitus; HAPO, The Hyperglycemia and Adverse Pregnancy Outcome observational study; IADPSG, The International Association of Diabetes and Pregnancy Study Groups; NDDG, The National Diabetes Data Group criteria; NICU, Admission to the neonatal intensive care; OGTT, Oral glucose tolerance test; OR, Odds ratio

\section{Background}

The prevalence of gestational diabetes mellitus (GDM) ranges between 0.7 and $10.1 \%$ according to different diagnostic criteria worldwide [1-4]. Although the early detection of GDM is important for reducing adverse pregnancy outcome, its diagnosis remains controversial [5-8]. In 2001, the American College of Obstetricians and Gynecologists (ACOG) recommended a two-step approach: a 50-g, 1-h glucose challenge test (GCT) and a 100-g, 3-h oral glucose tolerance test (OGTT) for pregnant women with a positive GCT [9]. Two diagnostic criteria, the National Diabetes Data Group (NDDG) criteria [10] and the Carpenter-Coustan (CC) criteria [11] for the 100 -g OGTT could be used. In our hospital, GDM is diagnosed according to the NDDG criteria when two or more plasma glucose levels exceed or are equal to 105, 190, 165, and $145 \mathrm{mg} / \mathrm{dL}$ for the fasting, 1-, 2- and 3-h plasma glucose tests, respectively [10]. The glucose levels described in the CC criteria are $95,180,155$, and $140 \mathrm{mg} / \mathrm{dL}$ for the fasting, 1-, 2- and 3-h plasma glucose tests, respectively [11].

In 2010, the International Association of Diabetes and Pregnancy Study Groups (IADPSG) developed new diagnostic criteria that are based on the Hyperglycemia and Adverse Pregnancy Outcome (HAPO) [5, 6]. The ACOG subsequently reaffirmed its recommendation of the twostep approach because of concerns about a new one-step approach, which showed no evidence of improving pregnancy outcomes and would significantly increase health care costs [7, 12]. Recently, ADA indicated that GDM screening can be accomplished using either of two strategies: the one-step 2-h 75-g OGTT or the two-step approach with a 1-h 50-g GCT followed by a 3-h 100-g OGTT for those who were GCT-positive.

Despite the different recommendations for the diagnostic threshold of GDM, the two-step approach remains commonly used worldwide. However, both the NDDG and CC criteria have been used, and the ACOG has not recommended either set of criteria over the other [13]. The National Institutes of Health (NIH) consensus development conference also recommends the use of the two-step approach with a 100-g OGTT using the CC or NDDG criteria [14].

Recent studies have attempted to determine whether the use of CC criteria or NDDG criteria affect pregnancy outcome but showed inconsistent results regarding the risk of adverse pregnancy outcome between the NDDG and CC criteria [15-20]. Additional file 1: Table S1 is a brief summary of these results. Thus, it was important to determine and understand the prevalence and risk for pregnant women with GDM who were diagnosed based only on the CC criteria (not on the NDDG criteria). Therefore, our main hypothesis was that the risk of adverse pregnancy outcome in women who were diagnosed with GDM based only on the CC criteria but not meeting the NDDG criteria was significantly higher than the risk in women who screened as negative based on the GCT. The second hypothesis was that there was an increased risk of adverse pregnancy outcome in women with GDM according to the NDDG criteria and that there was no difference in women with false-positive screening compared to women with negative 50-g GCT results.

\section{Methods}

\section{Study participants and data collection}

This retrospective cohort study collected laboratory data and medical records from pregnant women who were administered a 50-g GCT at 24 to 28 weeks of gestation and were delivered at the Ditmanson Medical Foundation Chia-Yi Christian Hospital (DMF-CYCH) between March 2006 and January 2013. Women with multifetal pregnancies, pre-existing diabetes, and pre-existing hypertension were excluded. This study was approved by the Institutional Review Board of the DMF-CYCH (CYCH IRB No: 100006). Plasma glucose levels were measured using a Hitachi 7170 automatic analyzer (Hitachi Co., Tokyo, Japan) at the DMF-CYCH central laboratory according to a standard clinical protocol.

\section{Two-stage approach for GDM screening and the classification of study participants}

The study included all women who underwent the twostep approach for screening and diagnosing GDM during the study period. The GCT was considered negative if the screening value was $<140 \mathrm{mg} / \mathrm{dL}$. If the GCT was positive, the women subsequently underwent a 100-g, 3-h OGTT to confirm GDM. Study subjects were classified as follows: (1) negative screening group: women with negative GCT 
results, (2) false-positive screening group: women with positive GCT results and negative 100-g OGTT results according to both CC and NDDG criteria, (3) CC-onlyGDM group: women with positive GCT results plus GDM diagnosis meeting the $\mathrm{CC}$ but not the NDDG criteria, and (4) NDDG-GDM group: women diagnosed with GDM using the NDDG criteria.

\section{Pregnancy outcomes: neonatal and maternal outcomes}

The measured pregnancy outcomes included adverse neonatal outcomes (including macrosomia ( $>4000 \mathrm{~g})$, preterm labor (delivery before 37 weeks), low birth weight $(<2500 \mathrm{~g})$, admission to neonatal intensive care (NICU), and Apgar scores $<7$ at 1/5 min) and maternal outcomes (including cesarean section, gestational hypertension or preeclampsia, shoulder dystocia, third- or fourth-degree perineal laceration, and postpartum hemorrhage). Gestational hypertension was defined as blood pressure $\geq 140 \mathrm{mmHg}$ systolic or $\geq 90 \mathrm{mmHg}$ diastolic after 20 weeks of gestation in a woman with previously normal blood pressure and blood pressure levels that returned to normal postpartum. Preeclampsia was characterized by gestational hypertension and proteinuria $(\geq 0.3 \mathrm{~g} /$ day or $\geq 1+$ on a urine dipstick) with or without pathologic edema [21].

\section{Statistical analysis}

In total, 9580 women and 11,468 pregnancies were enrolled in the study. Among the women, 1814 (18.9 \%) were recruited more than once because of multi-gravidity during the study period. Therefore, the difference among the glucose level groups was evaluated using the SAS SURVEYFREQ, SURVEYMEANS and SURVEYREG procedures. Data was clustered according to subject, and the variance of proportion was estimated using the Taylor series linearization method. The Rao-Scott chi-square test was used to test the categorical variables. The Wald F test was used for continuous data. Multiple mixed effects logistic regressions were used to determine the relationships between pregnancy outcome and glucose level group after adjusting for nulliparity, maternal age, body mass index (BMI) at delivery, and delivery year. In addition to the above confounding factors, delivery type (Cesarean section or not) was added and adjusted for the maternal outcome of postpartum hemorrhage. The associations found were described in terms of the adjusted odds ratio $(\mathrm{aOR})$ with a $95 \%$ confidence interval (CI). Logistic regression is widely used to adjust for confounders, not only in case-control studies, but also in cohort studies, yielding an aOR that approximates the adjusted relative risk when the disease is rare $(<10 \%$ incidence) [22, 23]. In addition, we reviewed previous studies that are listed in the PubMed and Cochrane databases; 6 large-sample size studies reported the issue, and 4 of these articles used multiple logistic regressions to estimate risk. For comparison, we used logistic rather than Poisson regression. A two-sided $p$ value $<0.05$ was considered statistically significant. All data were merged and analyzed using SAS 9.2 (SAS Institute, Cary, NC, USA). Our study adheres to the STROBE (Strengthening the Reporting of Observational Studies in Epidemiology) guidelines.

\section{Results}

A total of 12,274 pregnancies who received a GCT at 24 to 28 weeks of gestation and who delivered at DMF$\mathrm{CYCH}$ were enrolled. Among the cases, 289 women with multifetal pregnancies, pre-existing diabetes, and preexisting hypertension were excluded; information on height or weight at delivery was missing for 22 pregnancies. An additional 477 pregnancies were excluded from the analysis due to incomplete OGTT data. Thus, a total of 11,486 pregnancies were included in the study; of these, $9002(78.4 \%)$ screened negative, 1776 (15.5\%) screened false-positive, 251 (2.2\%) had CC-only-GDM, and 457 (4.0 \%) had NDDG-GDM (Fig. 1). The characteristics of

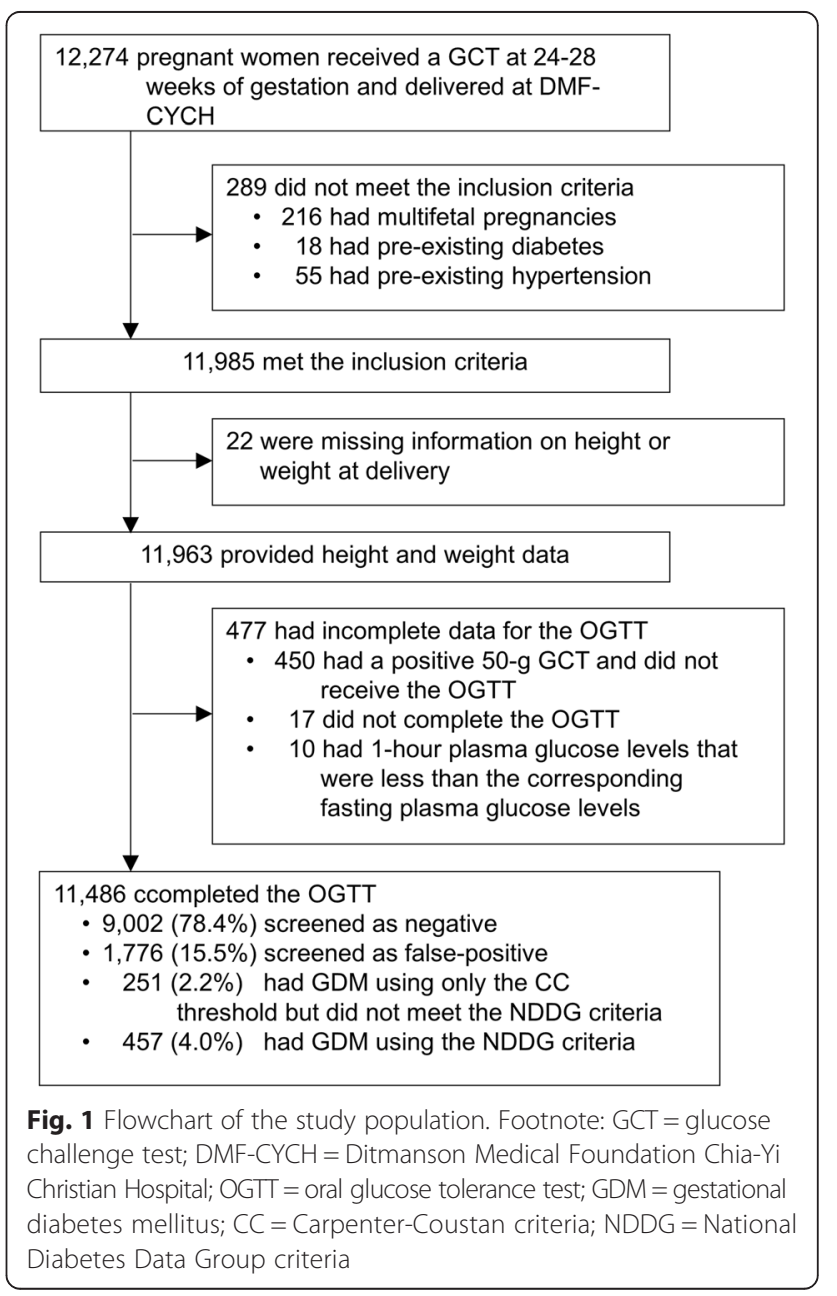


the study population among the 4 groups are shown in Table 1. The distributions differed among these groups.

Table 2 shows that the neonatal outcomes were significantly associated with the 4 groups, including macrosomia (0.8 to $3.5 \%, P<0.001$ ), preterm labor (6.5 to $12.3 \%$, $P<0.001$ ), low birth weight (5.6 to $9.0 \%, P=0.01$ ), and NICU admission (6.5 to $12.7 \%, P<0.001$ ); all of these conditions presented significantly higher incidence rates with higher glucose levels. The incidences of the maternal outcomes did not differ among the 4 glucose level groups, except for total cesareans (30.2 to $42.2 \%, P<0.001)$ and gestational hypertension or preeclampsia ( 2.8 to $7.0 \%, P<0.001$ ), for which the incidence increased with glucose level.

Table 3 shows the odds ratios of the outcomes after adjusting for confounding factors. Compared with the negative screening group, women with CC-only-GDM had significantly higher odds of macrosomia (aOR, 2.73; 95 \% CI, 1.18-6.31), low birth weight (aOR, 1.64; 95 \% CI, 1.01-2.64), and NICU admission (aOR, 1.61; $95 \% \mathrm{CI}$, 1.05-2.46). The NDDG-GDM group also showed significant results with greater aOR (95 \% CI): 3.15 (1.71-5.80), 1.81 (1.28-2.56), and 1.98 (1.47-2.68), respectively. The false-positive screening group did not differ from the negative screening group. Maternal outcomes did not significantly differ in the multivariable analyses, with the exceptions of gestational hypertension and preeclampsia.

\section{Discussion}

Our result was concordant with other studies that use of the CC criteria of 100-g OGTT in the two-step approach increased the prevalence of GDM. Previous studies comparing the NDDG and CC criteria were conducted in the Eastern U.S. [20], Western U.S. [16, 18], Canada [15], Spain [17], Turkey [24], and Taiwan [19]. The increase in prevalence of GDM in our study was similar to that found in two large sample studies $[16,18]$. The GDM prevalence increased from 31.8 to $125.7 \%$ (Additional file
1: Table S1) using the CC criteria compared with the NDDG criteria, possibly due to differences in ethnicity, lifestyle, or policies.

The findings of the study agreed with previous studies in that the additional patients that are diagnosed by CConly-GDM criteria are at higher risk of adverse pregnancy outcome $[15,16,18,20]$. In addition to fetal macrosomia, the pregnant women who were diagnosed based on CConly-GDM criteria were also at significantly increased risk of low birth weight and admission to NICU; these represent new findings (Additional file 1: Table S1). Our findings provide important additional information to the current debate about the diagnostic criteria for GDM regarding pregnancy outcome [25].

Although the incidence of macrosomia in the study was lower than that found in other studies [15-20], the rate remained significantly higher in the CC-only-GDM group than in the screening negative group. After adjusting for confounding factors, macrosomia remained a greater risk, consistent with the data reported by Berggren et al. [20]. However, a study in Spain did not yield significant findings [17].

For other neonatal outcomes, our results also showed strong evidence of significantly increased risks of low birth weight and admission NICU, and weak evidence of increased risk of preterm labor. Regarding the risk of NICU admission, two U.S. studies showed no association with the CC-only group [18, 20]; however, our data showed a significant association. Regarding low birth weight, the study of Berggren et al. showed no significant risk; however, our study showed a 1.6-fold higher risk compared to the screening negative group [20]. Regarding preterm labor, previous studies showed no significant association after adjusting for confounding factors $[17,18,20]$, with the exception of Hedderson et al. [16]. Our result regarding preterm labor was similar to that reported by Hedderson et al., and the adjusted odds ratios were $1.53(0.99-2.37)$ and 1.53 (1.16-2.03), respectively.

Table 1 Characteristics of the study population

\begin{tabular}{|c|c|c|c|c|c|}
\hline & Negative screening & False-positive screening & CC-only-GDM (not meeting NDDG) & NDDG-GDM & $P$ \\
\hline Number of observations & 9002 & 1776 & 251 & 457 & \\
\hline Number of clusters & 7640 & 1699 & 249 & 434 & \\
\hline \multicolumn{6}{|l|}{ Nulliparous status } \\
\hline Yes & $4627(51.4)$ & $936(52.7)$ & $115(45.8)$ & $212(46.4)$ & $0.03^{\mathrm{a}}$ \\
\hline No & 4375 (48.6) & $840(47.3)$ & $136(54.2)$ & $245(53.6)$ & \\
\hline Maternal age (years) & $29.0(28.9-29.1)$ & $30.6(30.4-30.8)$ & $32.1(31.5-32.6)$ & $32.5(32.0-32.9)$ & $<0.001^{\mathrm{b}}$ \\
\hline BMI at delivery $\left(\mathrm{kg} / \mathrm{m}^{2}\right)$ & $26.7(26.6-26.8)$ & $27.2(27.0-27.4)$ & $28.2(27.8-28.7)$ & $28.1(27.7-28.5)$ & $<0.001^{\mathrm{b}}$ \\
\hline 50-g GCT levels (mg/dL) & $110.4(110.0-110.7)$ & $158.0(157.3-158.7)$ & $164.8(162.4-167.3)$ & $179.7(177.0-182.3)$ & $<0.001^{\mathrm{b}}$ \\
\hline
\end{tabular}

Data are presented as means (95\% confidence interval) or $n$ (\%)

CC Carpenter-Coustan criteria, GDM gestational diabetes mellitus, NDDG National Diabetes Data Group criteria; BMI body mass index, GCT glucose challenge test ${ }^{a}$ The Rao-Scott chi-square test was performed using SAS SURVEYFREQ produces

${ }^{\mathrm{b}}$ ANOVA was performed using SAS SURVEYREG produces the Wald $\mathrm{F}$ test with their corresponding $p$-values 
Table 2 Pregnancy outcomes associated with the 4 glucose level groups

\begin{tabular}{|c|c|c|c|c|c|c|c|}
\hline Variable & Number & $\begin{array}{l}\text { Negative screening } \\
(n=9002)\end{array}$ & $\begin{array}{l}\text { False-positive screening } \\
(n=1776)\end{array}$ & $\begin{array}{l}\text { CC-only-GDM } \\
\text { (not meeting NDDG) } \\
(n=251)\end{array}$ & $\begin{array}{l}\text { NDDG-GDM } \\
(n=457)\end{array}$ & $\begin{array}{l}\text { Rao-Scott } \\
\text { Chi-Square }\end{array}$ & $P$ \\
\hline \multicolumn{8}{|l|}{ Neonatal outcome } \\
\hline Macrosomia (>4000 g) & $120 / 11,486$ & $75(0.8)$ & $22(1.2)$ & $7(2.8)$ & $16(3.5)$ & 39.0 & $<0.001$ \\
\hline Preterm labor (<37 weeks) & 789/11,486 & $581(6.5)$ & $127(7.2)$ & $25(10.0)$ & $56(12.3)$ & 27.0 & $<0.001$ \\
\hline Low birth weight (<2500 g) & $673 / 11,486$ & $507(5.6)$ & $105(5.9)$ & $20(8.0)$ & $41(9.0)$ & 10.9 & 0.01 \\
\hline Admission to $\mathrm{NICU}^{\mathrm{a}}$ & $789 / 11,471$ & $584(6.5)$ & $121(6.8)$ & $26(10.4)$ & $58(12.7)$ & 31.1 & $<0.001$ \\
\hline Apgar score $<7$ at $1 \mathrm{~min}$ & $127 / 11,486$ & $99(1.1)$ & $17(1.0)$ & $4(1.6)$ & $7(1.5)$ & 1.7 & 0.64 \\
\hline Apgar score $<7$ at $5 \mathrm{~min}$ & $37 / 11,486$ & $28(0.3)$ & $8(0.5)$ & - & $1(0.2)$ & - & - \\
\hline \multicolumn{8}{|l|}{ Maternal outcome } \\
\hline Cesarean section & $3632 / 11,486$ & $2721(30.2)$ & $617(34.7)$ & $101(40.2)$ & $193(42.2)$ & 46.3 & $<0.001$ \\
\hline $\begin{array}{l}\text { Gestational hypertension } \\
\text { or preeclampsia }\end{array}$ & $383 / 11,486$ & $255(2.8)$ & $82(4.6)$ & $14(5.6)$ & $32(7.0)$ & 38.6 & $<0.001$ \\
\hline Shoulder dystocia ${ }^{\mathrm{b}}$ & $79 / 7854$ & $64(1.0)$ & $7(0.6)$ & $3(2.0)$ & $5(1.9)$ & 5.5 & 0.14 \\
\hline $\begin{array}{l}\text { Third- or fourth-degree } \\
\text { perineal laceration }^{\mathrm{b}}\end{array}$ & $429 / 7854$ & $337(5.4)$ & $69(6.0)$ & $9(6.0)$ & $14(5.3)$ & 0.7 & 0.86 \\
\hline Postpartum hemorrhage & $98 / 11,486$ & $76(0.8)$ & $16(0.9)$ & $3(1.2)$ & $3(0.7)$ & 0.6 & 0.89 \\
\hline
\end{tabular}

Data are presented as $n$ (\%)

CC Carpenter-Coustan criteria, GDM gestational diabetes mellitus, NDDG National Diabetes Data Group criteria, NICU neonatal intensive care unit

The Rao-Scott Chi-Square test was performed

aExcluding neonatal death

${ }^{b}$ Only including vaginal delivery

In contrast, regarding adverse maternal outcomes, our results showed that Cesarean section, gestational hypertension and preeclampsia exhibited increasing incidence as glucose levels increased from the screening negative group to the NDDG-GDM group; however, the findings did not reach statistical significance in the CC-only-GDM group after adjusting for confounding factors. These results were different than those obtained in previous studies, which showed that the incidence of cesarean section $[15,16,18,20]$, gestational hypertension, and preeclampsia $[16,17,20]$ was higher in the CC-only-GDM group. We speculated that this result might have been influenced by

Table 3 Adjusted odds ratios for pregnancy outcomes

\begin{tabular}{|c|c|c|c|c|}
\hline Variable & $\begin{array}{l}\text { Negative screening } \\
(n=9002)\end{array}$ & $\begin{array}{l}\text { False-positive screening } \\
(n=1776)\end{array}$ & $\begin{array}{l}\text { CC-only-GDM (not meeting } \\
\text { NDDG) }(n=251)\end{array}$ & $\begin{array}{l}\text { NDDG-GDM } \\
(n=457)\end{array}$ \\
\hline \multicolumn{5}{|l|}{ Neonatal outcome } \\
\hline Macrosomia (>4000 g) & 1 & $1.30(0.79-2.15)$ & $2.73(1.18-6.31)^{c}$ & $3.15(1.71-5.80)^{c}$ \\
\hline Preterm labor (<37 weeks) & 1 & $1.09(0.89-1.34)$ & $1.53(0.99-2.37)$ & $1.90(1.39-2.58)^{c}$ \\
\hline Low birth weight $(<2500 \mathrm{~g})$ & 1 & $1.07(0.85-1.33)$ & $1.64(1.01-2.64)^{c}$ & $1.81(1.28-2.56)^{c}$ \\
\hline Admission to $\mathrm{NICU}^{a}$ & 1 & $1.02(0.83-1.25)$ & $1.61(1.05-2.46)^{c}$ & $1.98(1.47-2.68)^{c}$ \\
\hline Apgar score $<7$ at $1 \mathrm{~min}$ & 1 & $0.78(0.46-1.32)$ & $1.20(0.43-3.35)$ & $1.13(0.51-2.51)$ \\
\hline \multicolumn{5}{|l|}{ Maternal outcome } \\
\hline Cesarean section & 1 & $1.06(0.94-1.21)$ & $1.11(0.82-1.50)$ & $1.19(0.95-1.49)$ \\
\hline Gestational hypertension or preeclampsia & 1 & $1.36(1.04-1.79)^{c}$ & $1.42(0.79-2.58)$ & $1.70(1.11-2.60)^{c}$ \\
\hline Shoulder dystocia ${ }^{\mathrm{b}}$ & 1 & $0.61(0.27-1.34)$ & $1.95(0.59-6.46)$ & $2.02(0.78-5.23)$ \\
\hline Third- or fourth-degree perineal laceration ${ }^{b}$ & 1 & $1.04(0.78-1.37)$ & $1.05(0.52-2.14)$ & $0.98(0.55-1.74)$ \\
\hline Postpartum hemorrhage & 1 & $1.02(0.59-1.77)$ & $1.26(0.39-4.08)$ & $0.68(0.21-2.21)$ \\
\hline
\end{tabular}

Data are presented as adjusted odds ratios (95\% confidence intervals)

Odds ratios were adjusted for nulliparity, maternal age, body mass index at delivery, and delivery year. In addition to the above confounding factors, delivery type (Cesarean section or not) was added for adjustment based on the perinatal outcome of postpartum hemorrhage

CC Carpenter-Coustan criteria, GDM gestational diabetes mellitus, NDDG National Diabetes Data Group criteria, NICU neonatal intensive care unit

aEcluding neonatal death

bonly including vaginal delivery

${ }^{9} 95 \%$ confidence interval did not include 1 
factors such as ethnicity and the BMI variable. We used BMI at delivery to adjust the risk of pregnancy outcomes instead of the pre-pregnancy BMI, because the latter information was often missing in the database.

A similar study by Chou et al. in Taiwan enrolled 10,990 pregnancies in the urban area of northern Taiwan [19]. The prevalence of CC-only-GDM in this study and in our study was 4.4 and $2.2 \%$, respectively. The prevalence of NDDG-GDM in this study and in our study was 4.0 and $3.5 \%$, respectively. These findings demonstrated that women with GDM based on the CC criteria but not based on the NDDG criteria experienced a significant increase in macrosomia compared with women without GDM (4.5 vs. $2.3 \%, P<0.05)$. Other outcomes showed negative findings. Although both studies were conducted in Taiwan, the urban area (northern Taiwan) studied by Chou was different from the area examined in our study, which enrolled pregnant women in a rural area in southern Taiwan. Environmental factors, nutritional habits and socioeconomic status are therefore potential confounding factors. Furthermore, the maternal age and gestational age at delivery in Chou's study were different than in the current study $(32.0 \pm 0.4$ vs. $29.5 \pm 4.6$ years old; $38.5 \pm 0.1$ vs. $38.3 \pm 1.4$ gestational weeks).

Recent studies have focused on the new IADPSG criteria, which have further broadened the diagnostic criteria and stimulated debate about their cost-effectiveness [24, 26-28]. The latest ACOG practice bulletin clearly supports the two-step approach because the new IADPSG criteria would significantly increase health care costs and recommends that before the testing approach and diagnostic criteria for GDM are changed, the implications of such changes should be studied [12]. We have used the two-step approach for several years, and $6.2 \%$ of GDM according to the $\mathrm{CC}$ is appropriate and available for all populations that are covered by National Health Insurance. If the NDDG criteria were replaced by the CC criteria, the number of pregnant women diagnosed with GDM would increase by half, apparently increasing the burden of prenatal care. However, the higher cost of adverse neonatal outcomes on health care and society might be reduced, including long-term poor outcomes such as type 2 diabetes mellitus and the risk of childhood obesity and abnormal glucose metabolism [29-31]. The costeffective estimation of such potential outcomes warrants further study.

The study had several limitations; first, to the study was a single-hospital retrospective study. Therefore, the results obtained for Taiwanese women in the current study should not be generalized. The existing evidence is insufficient to build consensus regarding the diagnostic criteria for GDM on pregnancy outcome [25, 32, 33]. Further prospective studies and reviews are needed. Second, it would be interesting to expand our analyses by applying the IADPSG criteria to those who screened as positive and did not meet the NDDG criteria. However, because IADPSG uses a one-step approach requiring the full OGTT for the entire study population, we have no data regarding the ideas in the current study. Despite these limitations, the large sample of Asian women involved represented a major strength of our study.

\section{Conclusions}

Our findings suggested that diagnosing GDM according to the less strict CC criteria would detect a greater number of adverse neonatal outcomes. Women who met the CC criteria but not the NDDG criteria as well as women who met the NDDG criteria were at greater risk for macrosomia, low birth weight, and admission to the NICU compared with women who screened as negative. This evidence adds important information to the current debate regarding the diagnostic criteria for GDM on pregnancy outcomes.

\section{Additional file}

Additional file 1: Table S1. Comparison of our results with other studies. (DOC $67 \mathrm{~kb}$ )

\section{Acknowledgements}

We thank Chi-Chen Lin and Chung-Hsien Li of the Department of Information Technology at DMF-CYCH for their assistance with data acquisition. We also thank Dr. Lung-Chang Chien of the Department of Biostatistics at the University of Texas School of Public Health at the San Antonio Regional Campus for advice regarding statistics.

\section{Authors' contributions}

All authors (MCL, SSH, YHY, PW) contributed to the study design and data interpretation. $\mathrm{MCL}$ performed the data analysis. $\mathrm{MCL}, \mathrm{YHY}$ drafted the manuscript. All authors ( $\mathrm{MCL}, \mathrm{SSH}, \mathrm{YHY}, \mathrm{PW})$ critically revised the manuscript and approved the final version.

\section{Competing interests}

The authors declare that they have no competing interests.

\section{Author details}

${ }^{1}$ Department of Medical Research, Kuang Tien General Hospital, Taichung, Taiwan. ${ }^{2}$ Department of Obstetrics and Gynecology, Ditmanson Medical Foundation Chia-Yi Christian Hospital, 539 Chung-Shau Road, Chia-Yi City 600 , Taiwan. ${ }^{3}$ Department of Internal Medicine, Kuang Tien General Hospital, Taichung, Taiwan. ${ }^{4}$ Institute of Occupational Medicine and Industrial Hygiene, College of Public Health, National Taiwan University, Taipei, Taiwan.

${ }^{5}$ Department of Nutrition and Institute of Biomedical Nutrition, Hung Kuang University, Taichung, Taiwan.

Received: 14 January 2014 Accepted: 15 August 2016

Published online: 17 August 2016

\section{References}

1. Ferrara A. Increasing prevalence of gestational diabetes mellitus: a public health perspective. Diabetes Care. 2007;30 Suppl 2:S141-6.

2. Pedula KL, Hillier TA, Schmidt MM, Mullen JA, Charles MA, Pettitt DJ. Ethnic differences in gestational oral glucose screening in a large US population. Ethn Dis. 2009;19(4):414-9.

3. Fadl HE, Ostlund IK, Magnuson AF, Hanson US. Maternal and neonatal outcomes and time trends of gestational diabetes mellitus in Sweden from 1991 to 2003. Diabet Med. 2010;27(4):436-41. 
4. Zhang F, Dong L, Zhang CP, Li B, Wen J, Gao W, Sun S, Lv F, Tian H, Tuomilehto J, et al. Increasing prevalence of gestational diabetes mellitus in Chinese women from 1999 to 2008. Diabet Med. 2011;28(6):652-7.

5. International Association of Diabetes and Pregnancy Study Groups Consensus Panel MB, Gabbe SG, Persson B, et al. International Association of Diabetes and Pregnancy Study Groups Recommendations on the Diagnosis and Classification of Hyperglycemia in Pregnancy. Diabetes Care. 2010;33(3):676-82.

6. Metzger BE, Lowe LP, Dyer AR, Trimble ER, Chaovarindr U, Coustan DR, Hadden DR, McCance DR, Hod M, McIntyre HD, et al. Hyperglycemia and adverse pregnancy outcomes. N Engl J Med. 2008;358(19):1991-2002.

7. Gynecologists TACOOa. Committee opinion no. 504: Screening and diagnosis of gestational diabetes mellitus. Obstet Gynecol. 2011;118(3):751-3.

8. Association AD. Diagnosis and Classification of Diabetes Mellitus. Diabetes Care. 2011;34:562-9

9. Gynecologists TACOOa. ACOG Practice Bulletin. Clinical management guidelines for obstetrician-gynecologists. Number 30, September 2001 (replaces Technical Bulletin Number 200, December 1994). Gestational diabetes. Obstet Gynecol. 2001;98(3):525-38.

10. National Diabetes Data Group. Classification and diagnosis of diabetes mellitus and other categories of glucose intolerance. Diabetes. 1979;28(12):1039-57.

11. Carpenter MW, Coustan DR. Criteria for screening tests for gestational diabetes. Am J Obstet Gynecol. 1982;144(7):768-73.

12. Gynecologists TACOOa. Practice bulletin no. 137: gestational diabetes mellitus. Obstet Gynecol. 2013;122(2 Pt 1):406-16.

13. American Diabetes Association. Diagnosis and classification of diabetes mellitus. Diabetes Care. 2014;37 Suppl 1:\$81-90.

14. Vandorsten JP, Dodson WC, Espeland MA, Grobman WA, Guise JM, Mercer BM, Minkoff HL, Poindexter B, Prosser LA, Sawaya GF, et al. NIH consensus development conference: diagnosing gestational diabetes mellitus. NIH Consens State Sci Statements. 2013;29(1):1-31.

15. Naylor CD, Sermer M, Chen E, Sykora K. Cesarean delivery in relation to birth weight and gestational glucose tolerance: pathophysiology or practice style? Toronto Trihospital Gestational Diabetes Investigators. JAMA. 1996;275(15):1165-70

16. Hedderson MM, Ferrara A, Sacks DA. Gestational diabetes mellitus and lesser degrees of pregnancy hyperglycemia: association with increased risk of spontaneous preterm birth. Obstet Gynecol. 2003;102(4):850-6.

17. Ricart W, Lopez J, Mozas J, Pericot A, Sancho MA, Gonzalez N, Balsells M, Luna R, Cortazar A, Navarro P, et al. Potential impact of American Diabetes Association (2000) criteria for diagnosis of gestational diabetes mellitus in Spain. Diabetologia. 2005;48(6):1135-41.

18. Cheng YW, Block-Kurbisch I, Caughey AB. Carpenter-Coustan criteria compared with the national diabetes data group thresholds for gestational diabetes mellitus. Obstet Gynecol. 2009;114(2 Pt 1):326-32.

19. Chou CY, Lin CL, Yang CK, Yang WC, Lee FK, Tsai MS. Pregnancy outcomes of taiwanese women with gestational diabetes mellitus: a comparison of Carpenter-Coustan and National Diabetes Data Group criteria. J Womens Health (Larchmt). 2010;19(5):935-9.

20. Berggren EK, Boggess KA, Stuebe AM, Jonsson Funk M. National Diabetes Data Group vs Carpenter-Coustan criteria to diagnose gestational diabetes. Am J Obstet Gynecol. 2011;205(3):253 e251-257.

21. Gynecologists ACOOa. ACOG practice bulletin. Diagnosis and management of preeclampsia and eclampsia. Number 33, January 2002. Obstet Gynecol. 2002;99(1):159-67.

22. Zhang J, Yu KF. What's the relative risk? A method of correcting the odds ratio in cohort studies of common outcomes. JAMA. 1998;280(19):1690-1.

23. Hosmer DW, Lemeshow S. Applied logistic regression. New York: Wiley; 1989.

24. Kosus A, Kosus N, Turhan NO. Gestational diabetes: comparision of the carpenter and the coustan thresholds with the new thresholds of Turkish women and implications of variations in diagnostic criteria. J Matern Fetal Neonatal Med. 2012;25(6):616-22.

25. Hartling L, Dryden DM, Guthrie A, Muise M, Vandermeer B, Donovan L. Diagnostic thresholds for gestational diabetes and their impact on pregnancy outcomes: a systematic review. Diabet Med. 2014;31(3):319-31.

26. Langer O, Umans JG, Miodovnik M. The proposed GDM diagnostic criteria: a difference, to be a difference, must make a difference. J Matern Fetal Neonatal Med. 2013;26(2):111-5.

27. Reece EA, Moore T. The diagnostic criteria for gestational diabetes: to change or not to change? Am J Obstet Gynecol. 2013;208(4):255-9.
28. Duran A, Sáenz S, Torrejón MJ, Bordiú E, del Valle L, Galindo M, Perez N, Herraiz MA, Izquierdo N, Rubio MA, et al. Introduction of IADPSG Criteria for the Screening and Diagnosis of Gestational Diabetes Mellitus Results in Improved Pregnancy Outcomes at a Lower Cost in a Large Cohort of Pregnant Women: The St. Carlos Gestational Diabetes Study. Diabetes Care. 2014;37(9):2442-50.

29. Baptiste-Roberts K, Nicholson WK, Wang NY, Brancati FL. Gestational diabetes and subsequent growth patterns of offspring: the National Collaborative Perinatal Project. Matern Child Health J. 2012;16(1):125-32.

30. Metzger BE. Long-term outcomes in mothers diagnosed with gestational diabetes mellitus and their offspring. Clin Obstet Gynecol. 2007;50(4):972-9.

31. Langer $\mathrm{O}$, Umans JG, Miodovnik M. Perspectives on the proposed gestational diabetes mellitus diagnostic criteria. Obstet Gynecol. 2013;121(1):177-82.

32. Kanguru L, Bezawada N, Hussein J, Bell J. The burden of diabetes mellitus during pregnancy in low-and middle-income countries: a systematic review. Glob Health Action. 2014;7:23987.

33. Schneider S, Bock C, Wetzel M, Maul H, Loerbroks A. The prevalence of gestational diabetes in advanced economies. 2012.

\section{Submit your next manuscript to BioMed Central and we will help you at every step:}

- We accept pre-submission inquiries

- Our selector tool helps you to find the most relevant journal

- We provide round the clock customer support

- Convenient online submission

- Thorough peer review

- Inclusion in PubMed and all major indexing services

- Maximum visibility for your research

Submit your manuscript at www.biomedcentral.com/submit
(O) BioMed Central 